\title{
Electromagnetic Actuator for Tactile Communication
}

\author{
Basil Duvernoy ${ }^{1}$, Ildar Farkhatdinov ${ }^{2}$, Sven Topp ${ }^{3}$, and Vincent Hayward ${ }^{4,5,6}$ \\ 1 Sorbonne Université, Institut des Systèmes Intelligents et de Robotique, 75005 Paris, France \\ duvernoy@isir.upmc.fr \\ 2 School of Electronic Engineering and Computer Science, Queen Mary University of London, \\ London E1 4FZ, UK \\ 3 School of Psychology, The University of Sydney, NSW 2006, Australia \\ 4 Institute of Philosophy, School of Advanced Study, University of London, London, UK \\ 5 Actronika SAS, Paris, France \\ 6 ORCID 0000-0002-2102-1965
}

\begin{abstract}
Fingerspelling is a tactile code that enables linguistic communication with people who are Deafblind. We describe and undertake initial testing of a crucial component of a device that is designed to perform tactile fingerspelling with the speed and the clarity approaching that of a human signer. The component in question is a tactile actuator, which is based on a conventional electromagnetic motor, but which is carefully configured to meet the requirements of communication by tactile spelling. The actuator is intended to be easy to manufacture, reliable, inexpensive, to be made in many variants and to be safe to use.
\end{abstract}

Keywords: fingerspelling, tactile displays, actuators

\section{Introduction}

The practical realisation of tactile displays has long been a challenge. One factor which is frequently cited is the lack of availability of actuators that are sufficiently small, fast, strong, reliable, cost effective and have other desirable application-specific properties. In the past fifty years many tactile display designs have been described but are not surveyed here because numerous review articles are readily available and continue to be published at a steady pace. A result of the lack of adequate actuators is that today, with the exception of refreshable Braille displays, most commercially or pre-commercially available (i.e. evaluation kits) devices are single or few actuator systems. Multiple actuator tactile displays typically remain laboratory prototypes. From this perspective the current situation is not much different from that in 1927, see [2, Fig. 3-4].

\subsection{Why the development of purpose-specific tactile actuators is needed}

These observations justify the consideration of tactile displays, like R. H. Gault did almost a century ago, from the view point of narrowly defined use-cases. In this article, we focus on one such single use-case: The stimulation that is necessary to bring about linguistic communication by touch. One mode of tactile communication that enjoys widespread practice, besides Braille text reading, is the family of signing and spelling 
languages that is utilised by the Deafblind community. This community comprises hundreds of thousands of individuals in Europe (349,000 individuals in the UK alone with an estimated population density of 1:250 [5]). The primary form of communication within the Deafblind domain is reliant of the sense of touch but may also include auditory or visual codes (dependent on visual and auditory acuity). For reasons that range from aetiology to life opportunities, approximately a quarter of this community communicates linguistically by placing tactile symbols into the palm of the hand. The development of a technology capable of conveying the relevant tactile codes rapidly and clearly would nevertheless be worthwhile, provided of course, that the technology would be economically viable.

\subsection{Brief Introduction to Tactile Spelling}

Due to isolation of individuals with Deafblindness, methods of tactile communication have evolved separately within countries and historically across continents and subcontinents. The category of interest here is that of the tactile fingerspelling codes (as opposed to hands-on-signing) that are used in the British Commonwealth, Italy, Germany and several other countries. These codes are signed by touching the sensitive inside region of the hand and represent a letter, word, concept or even an entire phrase. A significant downside of tactile fingerspelling is the ergonomic discomfort and biomechanical stress to which tactile signers are subjected with prolonged signing leading to Repetitive Stress Injuries (RSI) in the fingers, wrist and shoulders. At its core, fingerspelling is based on brief finger tapping actions and swift swiping movements on the palm to sign letters and numbers. Figure 1 illustrates the basis of the UK and Australian system. Some code extensions also use secondary interactions such as finger tap closely followed by a finger 'flick'.

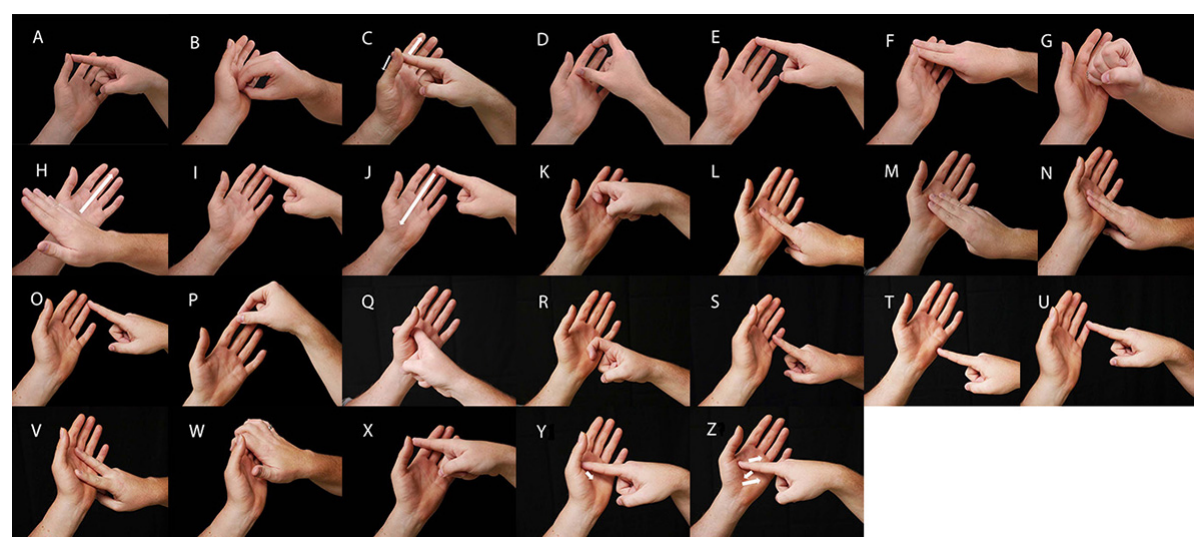

Fig. 1. Alphabet coding of British the "Deafblind Manual" and the Australian "Deafblind Tactile Fingerspelling". 
One should not assume, however, that fingerspelling, like Braille code, can only encode letters and numbers. The reality is that its expressiveness is akin to that of auditory and visual codes. This fact is particularly true of Deafblind Tactile Fingerspelling, a system developed in Australia and which at the time of writing is the primary focus of our attention $[3,6]$. There is prosody, emphasis, and other forms of modulation that are like those of speech and that operate at the lexical, grammatical, semantic, and emotional levels, in addition to the use of non-lexical short-hand symbols, abbreviations, smileys, geometric figures, directional cues, nicknames among others. Useful tactile communication technologies should account for, reproduce, and represent these natural forms of modulation.

An observation which is highly relevant to design of actuators specifically tailored for tactile communication through fingerspelling is the fact that professional tactile interpreters can sign continuously at a rate of up to five symbols per second. To estimate the speed requirement of a tactile display capable of reproducing natural signing, an informal test was performed with the help of a volunteering professional interpreter. The interpreter was asked to sign the pangramic sentence "The quick brown fox jumps over the lazy dog". A total of nine seconds was required to convey thirty-five letters. A breakdown of this sentence by symbol type indicates that six were signed by sliding, fifteen were signed with a single tap, fourteen by place-and-hold, and one by a pinch. Rough estimates suggest that symbols requiring swiping can take up to $300 \mathrm{~ms}$ in duration while the much shorter tapping motions are executed under $30 \mathrm{~ms}$. While this test was conducted at the maximum speed of the interpreter it should be noted that this pace cannot be comfortably sustained for more than a few minutes.

Before we review the requirements for an actuator suitably designed for tactile fingerspelling, the complete device is now briefly described. This device will be more fully described and evaluated in future publications.

\subsection{Brief Overall Device Description}

The design philosophy follows from the need to provide a device capable of supporting prolonged use while not impeding the freedom of movement of the person using it. It is evident that in actual practice, the hand of a user may be called upon at a moment's notice to tasks other than receiving tactile code. These basic, pragmatic requirements disqualify approaches based on "tactile gloves" which are impractical to don and doff, and which cause discomfort and sweating after a few minutes of use. Moreover, tactile gloves fail at providing clean channel separation during stimulus generation - a requirement that is essential for intelligibility. Human hands are sensitive to vibrations of very low amplitude, viz. of one micrometer or less. Attaching actuators to textiles in continuous contact with the skin cause mechanical signals to propagate away from the locus of stimulation, irremediably blurring the signals being transmitted.

We developed instead a hand interface that comprises two components. There is an array of twenty-four actuators (this number is to be justified in future publications), see Fig. $2 \mathrm{~s}$, which are distributed in three dimensions to maximise their mutual distances ensuring a minimum of mechanical and magnetic interference between them. The individual actuators are held inside pods and are precisely positioned laterally. The moving 

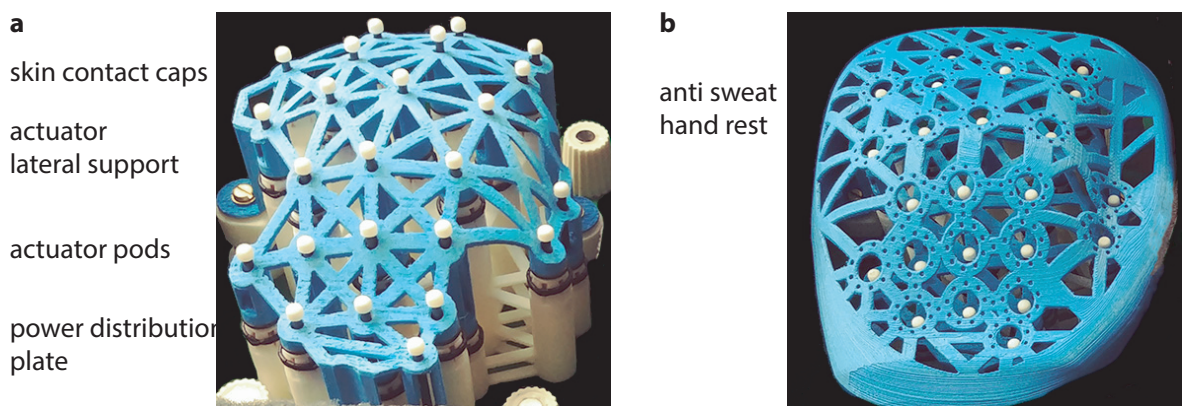

Fig. 2. Whole-hand tactile display. a. Array of twenty four actuators housed in individual pods. b. View of the device with the hand rest shell positioned above the array without direct contact.

parts of the actuators, termed 'plungers', drive vertical rods that terminate with customisable, skin-contacting caps. The rods transmit movement through loose tolerance guidance, thus operating with little friction and without special guiding elements.

The actuator array is covered by a hand-supporting shell that serves a triple purpose. It mechanically isolates the hand from the actuator array with the result that tactile stimulation takes place only when and where actuators are activated. When not used, the skin contacting caps are recessed and parasitic vibrations are not transmitted to the hand at undesired loci of the volar region of the hand. In other words, only the activated actuators stimulate the skin at a specific locations, and the signals do not leak elsewhere. The second purpose of the shell is to support the hand against gravity, a key requirement for prolonged use. The third purpose is to provide for a removable interface that can be cleaned and can be customised.

\subsection{Summary of Requirements}

We can now specify more accurately the desired properties of suitable actuators. Ten requirements are weighted equally and must be met without exception, namely,

1. to be small enough to accommodate twenty to thirty actuators in a space delimited by a human hand above a table;

2. to be capable of delivering impacts on the hand similar in nature to those made by human fingers. The energy dissipated by such impacts may be estimated at $0.2 \mathrm{~mJ}$. This figure can be worked out from acceleration data from human fingers [1];

3. to achieve repeating these impacts at a rate of at least four per second distributed over the device. Here, the performance of the transducer shouldn't be specified with respect to the perceptual performance of the receiver, but rather with respect to the motor performance of signers. Most experienced Tactile Interpreters can finger tap three times per second on average [4];

4. to assume a recessed position when not in use to avoid unwanted parasitic stimulation, even when not activated;

5. to provide vibro-tactile stimulation in the range $100-500 \mathrm{~Hz}$ such that frictional noise can be simulated with the aim of producing good apparent motion, a commonly accepted range of frequencies [7]; 
6. to be safe to use and to interact with, even in the case of malfunction;

7. to be easy to be manufactured and replicated given basic technical skills, and operate at nominal performance even with low tolerance manufacturing;

8. to have a moderate cost and to rely on widely available materials and components;

9. to be easily maintainable, interchangeable, and if possible washable;

10. and to be reliable, maintainable, and support millions of cycles before failure.

\section{Motor Design and Realisation}

To achieve the stated objectives, it became apparent early in the design process that the guiding mechanism on which the moving parts relied would be the weak point of any design. From this observation, it was concluded that vertical displacement would be a primary constraint owing to its simplicity. Vertical displacement can be achieved with no other mechanism than a plunger moving in a vertical bore. The second, early decision concerned the type of primary actuation. Unless shown otherwise, the electrodynamic motor which vastly dominates the transducer industry, was the only available option. Any of other known modes of realisation of actuation failed to meet one or several of the constraints outlined in Section 1.4. Design iterations, using well-known principles of realisation of electrodynamic motors [8], rapidly converged to a design based on an axial organisation of the magnets. The presently realised motor is represented in Fig. 3a.
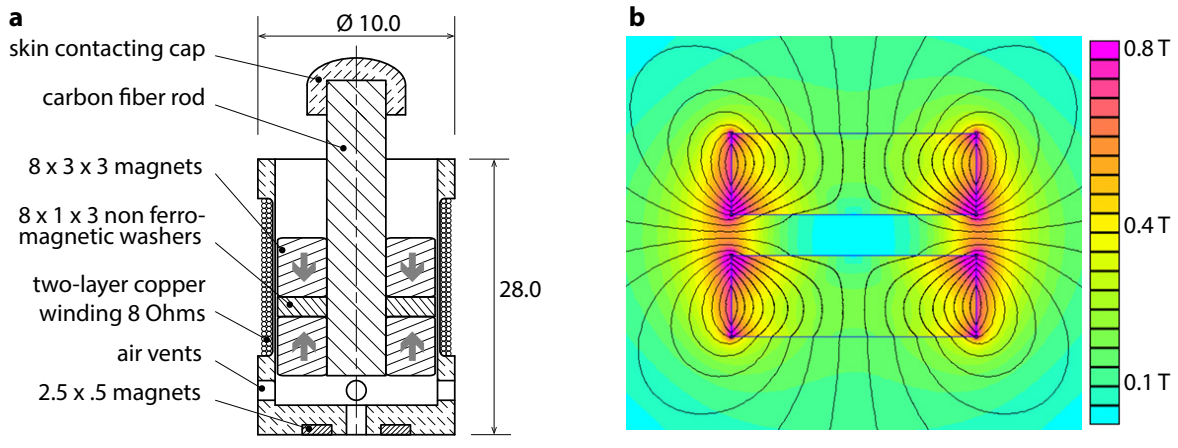

Fig. 3. Actuator Design. a. Grey arrows indicate the magnet polarities. b. Dimensional optimisation of the magnetic circuit.

The motor comprises only two subassemblies. The first is a thin-walled mandrel on which a coil is wound. The mandrel includes thicker walls at either end to increase its structural strength. The second is a moving subassembly comprises two axially polarised ring magnets secured around a non-ferromagnetic rod. It is made of a carbon fibre composite but this is not essential. The moving subassembly acts like a plunger which is inserted in the bore of the coil subassembly, remaining there under the action of gravity, and is free-moving. The present design produces a nomimal force of approximately $0.15 \mathrm{~N}$, meaning that for a plunger mass of about $3 \mathrm{~g}$, it is capable of an acceleration five times that of gravity. 
Recal that the central design goal was to provide for two modes of stimulation. The first mode is an impulsive mode aimed at reproducing the impact of a finger and the second is aimed at providing for vibro-tactile stimulation in an intermittent fashion.

\subsection{Impact Mode}

An impact was achieved by simply pulsing current in the coil. The plunger shot up from which two scenarios can arise. If there is a hand in range of the motor - the pods and the hand rest shell were strategically designed to locate the skin within 3.0$4.0 \mathrm{~mm}$ of the cap in its rest position - , then a collision occurred. The sensation of impact was very similar to that generated by a tapping finger. If for any reason no hand was present to determine a collision, then the plunger continued to rise. However, see Fig. 3b, the magnetic field interfering with the coil soon reversed and so did the electrodynamic force, causing the plunger to be pushed back into the bore. If for any reason current should ceased, a hard stop prevented the plunger from exiting, at the cost of an unpleasant knocking noise. During normal operation, current timing and intensity could be judiciously selected to ensure silent operation of the motor.

It is important to recall that vibration-free operation was an essential requirement for intelligibility which required precise timing of current reversal to stop the plunger before striking the bottom of the bore. Precise timing could not be achieved without a complex closed-loop operation. A solution was to provide for passive braking when approaching the end of the downward movement by tiny magnets inserted at the bottom of the bore that interfered with the main magnets to create a repelling force of the form $1 / a^{2}$, where, $a$ is the magnet-to-magnet distance. A secondary braking mechanism was a viscous force caused by the flow of air through small vents that were calibrated to provide a desired amount of damping. Combining these elements makes it possible to determine the nonlinear equation governing the motor,

$$
m \ddot{z}(t)+b \dot{z}(t)+k(z) z(t)+m g=a(z(t), i(t)),
$$

where $m$ is the mass of the plunger, $z(t)$ is the vertical position of the plunger, $k(z)$ is a nonlinear spring modelling the action of the braking magnets, $g$ is the acceleration of gravity, and $a(z, i)$ models the variation of the electrodynamics force as a function of the plunger position, $z(t)$, and of the current, $i(t)$, flowing in the coils. The functions $k(z)$ and $a(z, i)$ were not empirically determined, owing to the final design of the actuator not being frozen. An interesting "sling-shot" effect involved the reversal of the current during an initial pulse followed by switching to a positive pulse in a second phase.

\subsection{Vibro-Tactile Mode}

While in this mode of operation, the motor was biased to cause the plunger to lift upward and push gently against the skin. Remaining in this position, the motor could be used as a wide-bandwidth electrodynamic transducer. In our present design the lifting and gentle push on the skin was achieved for only $10 \%$ of the nominal current capacity, allowing plenty of headroom for oscillatory stimulation. In this mode, we may model the actuator as a linear system working around a biased operating point,

$$
m \ddot{z}(t)+b \dot{z}(t)=c_{b}(i(t))+f(d, t),
$$


where, $c_{b}$ represents the motor's $B l$ product at the point of operation, where $f(d, t)$ is the dynamic model of the mechanical load given by a finger, and where $d$ stands for the surface deflection of the finger. Estimates for $f(d, t)$ can be found in [9].

\section{Fabrication}

Various dimensions and materials were optimised using CAD tools. In particular it was found that the magnet geometry indicated in Fig. 3 provided good performance with conventional, commercially available neodymium magnets. With some precautions, a 3D-printing process using fused deposition gave satisfactory manufacturing of the mandrel. The coil winding was initially undertaken manually. A desk-top winding machine was designed in-house to speedup the winding process and achieve tight and regular wire packing. It was found that an $8 \Omega$, two-layer coil gave a good compromise, similar to coils incorporated in audio loudspeakers. The magnets were bonded to stock carbon fibre rods cut at length. Not accounting for labor, an overall costing of the components for small scale production came to a total of $13 €(9 €$ for the mandrel and $4 €$ for the plunger). Large cost savings could certainly be obtained through bulk procurements.

\section{Evaluation}

\subsection{Time Domain, Large Signal Response}

This test was intended to verify that our actuator could effect rapid taps at speeds and intensities outlined in the requirements. The actuator was commanded with brief $20 \mathrm{~ms}$, $3.3 \mathrm{~V}$ pulses at $90 \mathrm{~ms}$ intervals and its movements were monitored using a miniature accelerometer (ADXL 335, Analog Device, PC-board-less custom commissioning, mass $50 \mathrm{mg}$ ). The response is in Fig. 4, showing that the motor was clearly capable of repeating ten taps per second. Figure $4 \mathrm{~b}$ shows that acceleration (smoothed by a second-order lowpass Butterworth filter) tracked the input command with some disturbances due to the frictional noise of the magnet sliding inside the bore. Figure 4a shows the numerically integrated raw acceleration signal that estimated, after de-trending, the plunger velocity and position. Decreasing velocities were when the plunger was falling back inside the bore under the action of gravity and increasing velocities is when it was propelled upward. It can be observed that the plunger velocity reached $0.4 \mathrm{~m} / \mathrm{s}$ when the vertical displacement was $4 \mathrm{~mm}$, leading to an inelastic impact energy of $0.24 \mathrm{~mJ}$.

\subsection{Frequency Domain, Small Signal Response}

We analysed the frequency response of the actuator by interposing the miniature accelerometer between the rod and the cap in contact with the finger of a volunteer, as in Fig. 5a. The motor was biased at $20 \%$ of its current capacity and a small oscillatory voltage was superposed to the bias. The actuator was driven with sinusoidal signals at frequencies ranging from $20 \mathrm{~Hz}$ to $1000 \mathrm{~Hz}$ (by steps of $10 \mathrm{~Hz}$ from 20 to $500 \mathrm{~Hz}$ and of $50 \mathrm{~Hz}$ between 500 and $1000 \mathrm{~Hz}$ ). The measured acceleration and input voltage recordings for each frequency were used to produce the frequency response shown in Fig. $5 \mathrm{~b}$ 


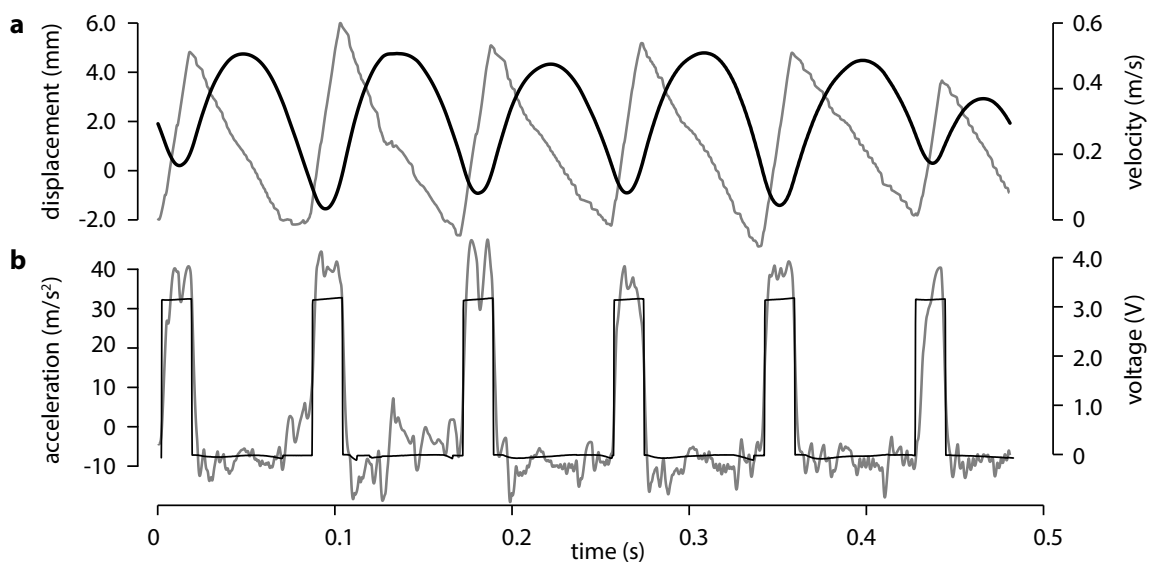

Fig. 4. Large signal, time domain performance. a. Estimated velocity (thin gray line) and position (black line) as a function time. b, Voltage input (thin gray line) and resulting acceleration (smoothed) starting at the value of negative acceleration of grativity.

using the Fast Fourier Transform function of Matlab. The plot demonstrates that for the range of frequencies $20-200 \mathrm{~Hz}$ the actuator can be modelled by a mass. The slight $\sim 3 \mathrm{~dB}$ drop around $250 \mathrm{~Hz}$ may be attributed to the viscous damping properties of the finger [9], while the rise in signal magnitude in the high frequencies can be explained by the increasing contribution of frictional noise in this range. This effect can be observed in Fig. $5 \mathrm{c}$ showing the noise-sensitive acceleration signal in the time domain.

a
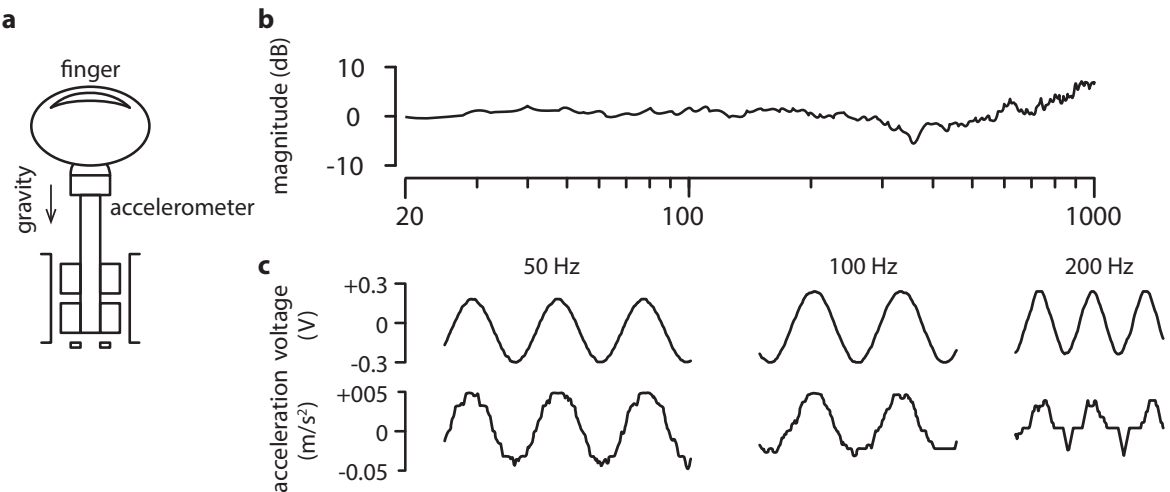

Fig. 5. Small signal performance. a. Measurement condition. b. Bode plot of the acceleration response. c. Time domain response at different frequencies. 


\section{Discussion / Conclusion}

We set out to design and produce an actuator suited to the purpose of generating the sensations required for the reproduction of tactile codes used in Deafblind Tactile Communication. Whilst we have no doubts that further iterations of design will be required, our initial investigations and characterisation of the motor provided promising results.

Its small overall size gives it the capacity to be utilised in a high-resolution displays. An array of twenty actuators spread over the sensitive area of the volar part of the hand is feasible for the greatest majority of hand sizes and shapes. In some cases, the capacity to create even denser groupings of actuators is a practical proposition.

The pricing of materials, of the manufacturing costs, and of the the complexity of production have all been kept to a minimum. Materials are easily sourced from the market and the possibility for cost reduction by a factor ten is quite achievable. Manufacturing costs and complexity are low with construction requiring little expertise other than care. The modular design means that, in the event of failure, actuators be rapidly reproduced and replaced, or stocked as spares.

On a mechanical level, the plunger component of the design can easily be seen meeting our requirement of being recessed when not in use. The impact capabilities could be classified as equivalent to, greater or less than those exerted by a human interpreter. Initial evaluations made by the third co-author, a Deafblind researcher, suggests that forces being exerted are in some cases too high for prolonged usage.

While it is noted that professional interpreters can sign at a speed of four symbols per second and we aimed to reproduce this performance. Our volunteering interpreter suggested that such speeds are impractical to maintain over extended periods of time. An estimates of two symbols per second is a more realistic base line. The proposed actuator design easily produced ten taps per second demonstrating a fair amount of performance headroom for more rapid contacts. Whether these higher speeds would be intelligible will require further investigation.

Our operating frequency range and quiet operation also meet initial requirements but may need some further refinement. Activation of the motor below $400 \mathrm{~Hz}$ frequencies appears to generate little or no noise. However, a noisy response was observed when the driving signals exceeded $400 \mathrm{~Hz}$. Possibilities for this inconvenience may be that the skin contacting cap decoupled from the skin or that the higher frequency ex-

cited a nonlinear clattering regime. Further investigations will be required to clarify and resolve this issue before rigorous human trials can be undertaken.

\section{Acknowledments}

The research reported herein is a component of a larger project which was, and is generously supported by three consecutive Google Faculty Awards to V.H. and S.T ("Tactile Communicator for Use by The Deafblind; Software Layers for Deafblind Tactile Communication; Hand-to-Hand Remote Deafblind Tactile Communication”). B.D. was awarded a post-graduate fellowship by the project SMART of the Faculté des Sciences de Sorbonne Université. 


\section{References}

1. Christou, E.A., Shinohara, M., Enoka, R.M.: Fluctuations in acceleration during voluntary contractions lead to greater impairment of movement accuracy in old adults. Journal of Applied Physiology 95(1), 373-384 (2003)

2. Gault, R.H.: "Hearing" through the sense organs of touch and vibration. Journal of the Franklin Institute 204, 329-358 (1927)

3. Johnston, T., Schembri, A.: Australian Sign Language (Auslan): An introduction to sign language linguistics. Cambridge University Press (2007)

4. Peters, M., Durding, B.M.: Handedness measured by finger tapping: A continuous variable. Canadian Journal of Experimental Psychology / Revue canadienne de psychologie experimentale 32(4), 257-261 (1978)

5. Robertson, J., Emerson, E.: Estimating the number of people with co-occurring vision and hearing impairments in the uk. Tech. Rep. 2010:1, CeDR, Lancaster University, UK (2010)

6. Topp, S.: Deafblind haptic speech, a vibrotactile device proposal. Master's thesis, School of Computer Science and Engineering, University of New South Wales, Australia (2013)

7. Verrillo, R.T.: Psychophysics of vibrotactile stimulation. Journal of The Acoustical Society of America 77(1), 225-232 (1985)

8. Wang, J., Jewell, G.W., Howe, D.: Analysis and design of tubular linear permanent magnet machines. IEEE Transactions on Magnetics 35(3), 1986-2000 (1999)

9. Wiertlewski, M., Hayward, V.: Mechanical behavior of the fingertip in the range of frequencies and displacements relevant to touch. Journal of Biomechanics 45(11), 1869-1874 (2012) 